\title{
Towards Social Mobile Blended Learning
}

\author{
Amr Abozeid ${ }^{1}$, Mohammed Abdel Razek ${ }^{1,2}$, Claude Frasson ${ }^{3}$ \\ ${ }^{1}$ Azhar university, Faculty of science, Math.\& Computer Science Depart. Naser City, Cairo, Egypt. \\ ${ }^{2}$ Deanship of Distance Learning, King Abdulaziz University, Kingdom of Saudi Arabia \\ ${ }^{3}$ Computer Science Department, University of Montréa,CP 6128 succ. Montréal, QC, Canada. \\ amrapozaid@gmail.com,maabdulrazek1@kau.edu.sa,frasson@iro.umontreal.ca
}

\begin{abstract}
Mobile technologies and Web 2.0 have led to explosions of communication, with a resulting increased need for people to process and utilize that new communication. This paper presents a new approach to create an integrated approach for learners by bring traditional education, mobile learning, along with social network into one adaptive blended learning environment. This approach introduces an adaptation mechanism to adapt learning objects to meet learner characteristics and their mobile capabilities.
\end{abstract}

Keywords: Social Network, Web 2.0, Mobile Learning, Adaptive Learning.

\section{Introduction}

Nowadays, the growth of online education encourages teachers in physical classrooms to use Internet-based content and resources. Meanwhile, mobile learning continues to challenge the boundaries imposed by traditional classroom learning to improve education and exploit technology in furthering that aim [4]. The need of (just in time - just enough) learning and the diversity of learners' characteristics as well as mobile technologies requires adaption for different cases [2].

This paper presents a framework for system architecture to combines the best elements of online, social network and face-to-face learning via mobile. It tends not only to create social blended learning environment, but also to adapt course's learning object. Our adaptive learning platform aims to build learning environment and provide learning objects based on learner's individualized information usage behavior, habits, preferences and etc [1]. The adaptation individualize the content based on learner's Level of knowledge (beginner, immediate, professional), learning styles (active - reflective, sensing - intuitive, visual - verbal, sequential - global), location (on campus, off campus), time (allowed time) [3].

In this paper, we display the architecture of Social Mobile Interactive Blended Learning System (SMIBLS). SMIBLS uses mobile and Bluetooth technologies to increase interactions and communications between instructors and students during classroom on campus, while, it uses Web 2.0 to enrich the communication between instructors and students during classroom off campus. MIBLS contains learning activities that leads to enhance learning process during classroom. As shown in Figure1, SMIBLS consists of two basic user interfaces: one devoted to the instructor and the other for the student. 
These interfaces can be accessed from Mobile and PC desktop as well. In order to provide adaptation, there are two modules: Learner Adaptation Module (LAM), and Instructor Adaptation Module (IAM). These modules use an adaptation engine to adapt the educational activity according to the context. LAM is the process of automatically adjusting learning contents based on learner's needs. The adaptation measures the needs based on the contexts which are considered by learners context (e.g. preferences, knowledge level and style), the educational context (e.g. requirements, pedagogical theory, achievements and results), the infrastructures context (e.g. networks, devices) and the environments context (e.g. neighbors, weather and noise level) [3]. Meanwhile, LAM allows user to collaborate via Web 2.0 tools like Face book and Twitter to establish a live communication between instructor in the classroom and his students outside the classroom.

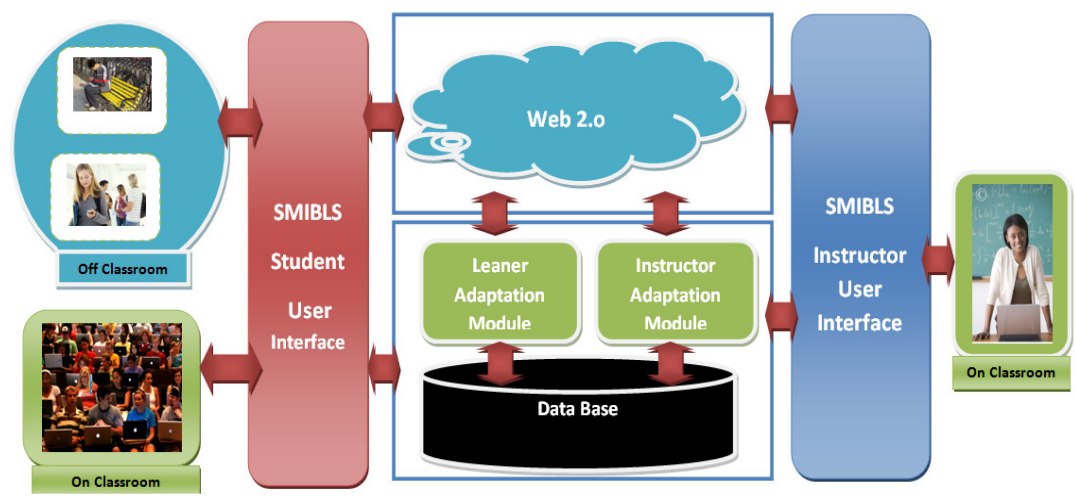

Figure 1 SMIBLS Architecture

IAM allows instructor to formulate a quiz or a question and send it to students in the classroom or to the student outside the classroom. IAM lets multimedia learning objects with different format such as swf, mp3 and mp4. Each LO is a file contains the learning content that cover part from the chapter objectives. The LO should be small and meaningful in order to enable students browsing it in their free time or bus traveling. From the education perspective, we suggested that LO should contain definitions, theories, remarks, and important parts covered in chapter or lecture notes.

\section{References}

1. Amr Abozeid, Mohamed A. Razek, Hosam Farouk El-Sofany, Fayed F. M. Ghaleb, Mobile Interactive Blended Learning System.IEEE Multidisciplinary Engineering Education Magazine.5,(2010).

2. Drira, R., et al.:What can we adapt in a Mobile Learning Systems?The Proceedings of the Second International Conference on Interactive Mobile and Computer Aided Learning (IMCL 2007).

3. Economides, Anastasios A:Adaptive context-aware pervasive and ubiquitous learning.International Journal of Technology Enhanced Learning.1,169--192 (2009).

4. Mohammed A. Razek, Hisham Jameel Bardesi: Towards Adaptive Mobile Learning System. IEEE-11th International Conference on Hybrid Intelligent Systems (HIS 2011): 493-498. 